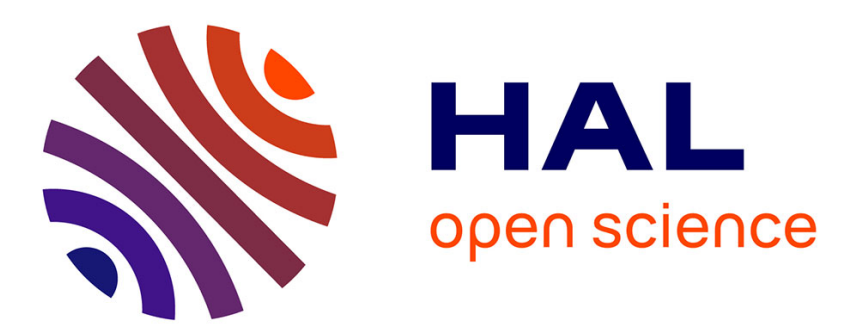

\title{
Professionnaliser le client: le travail du marché dans une entreprise de restauration collective
}

\author{
Christèle Dondeyne
}

\section{To cite this version:}

Christèle Dondeyne. Professionnaliser le client: le travail du marché dans une entreprise de restauration collective. Sociologie du Travail, 2002, 44 (1), pp.21-36. halshs-00626413

\section{HAL Id: halshs-00626413 \\ https://shs.hal.science/halshs-00626413}

Submitted on 11 Oct 2011

HAL is a multi-disciplinary open access archive for the deposit and dissemination of scientific research documents, whether they are published or not. The documents may come from teaching and research institutions in France or abroad, or from public or private research centers.
L'archive ouverte pluridisciplinaire HAL, est destinée au dépôt et à la diffusion de documents scientifiques de niveau recherche, publiés ou non, émanant des établissements d'enseignement et de recherche français ou étrangers, des laboratoires publics ou privés. 


\section{Professionnaliser le client : le travail du marché}

\section{dans une entreprise de restauration collective}

Professionalize clients : The work of the market in a catering firm

Christèle Dondeyne

Laboratoire d'économie et de sociologie du travail (Lest), CNRS, 35, avenue Jules-Ferry, 13626 Aix-en-Provence cedex, France

Résumé

Nous montrons dans cet article que si le client est bien au principe des stratégies de l'entreprise, la professionnalisation des clients est organisée par l'entreprise pour que ceux-ci prennent la mesure des actions qu'elle entreprend. La professionnalisation des clients mise en place dans une entreprise de restauration collective, Restocol, s'appuie sur une rationalisation des relations avec les clients et sur des innovations dans les prestations qui leur sont proposées. Elle est principalement opérée par les cadres par un travail d'argumentation, qui conduit à poser l'hypothèse de l'existence d'une technique des métiers relationnels.

\section{Abstract}

Catering for establishments is a fertile field for analyzing changes in the relations between the market and its organization. These relations were closely observed between the management of Restocol (a catering firm), its clients (medical establishments), and the persons who eat in the cafeterias. Although the company centers its strategies around its clients, it organizes for professionalizing them so that they understand its actions. This professionalization, as implemented in this catering firm, is based on a rationalization of relations with clients and on innovations in the services proposed to them. Since it is mainly the managers who do this through a work of argumentation, we can hypothesize that a technique exists of relational skills.

Mots-clé: Travail des cadres; Professionnalisation; Client; Marché; Rationalisation; Restauration collective

Keywords: White-collar work; Professionalization; Clients; Market; Rationalization; Cafeteria

1. La rationalisation des relations avec le client dans une entreprise de restauration collective ou l'émergence d'un nouvel ordonnancement productif 


\subsection{Le client dans la conduite de l'organisation}

\subsubsection{Une spécialisation par segments de clientèles}

\subsubsection{Transmettre des savoirs codifiés au client}

\subsubsection{Par des dispositifs spécifiques}

\subsubsection{Par des prestations qui révèlent ces savoirs}

\subsection{La progressive normalisation de l'organisation de la production}

2. La professionnalisation du client ou comment partager les règles de l'organisation

2.1. De l'hypothèse d'une socialisation au restaurant à l'hypothèse d'une professionnalisation du client par l'organisation

\subsection{Transmettre les règles de l'organisation au client}

2.2.1. Quand la professionnalisation du client marchand gagne du terrain: le travail des responsables de secteur

\subsubsection{Les employés de restaurant, entre le client et l'entreprise de restauration}

\subsection{Conclusion : les conditions d'une professionnalisation des clients}

\section{$\underline{\text { Références }}$}

Si aujourd'hui le marché subordonne plus qu'hier le travailleur et l'entreprise, le défi posé à la sociologie du travail est de l'intégrer dans ses analyses. La sociologie qui s'intéresse aux professionnels du marché désigne un objet, le travail marchand, qui porte sur la mise en forme de la relation marchande. La piste ainsi tracée peut être suivie à l'intérieur de l'entreprise lorsque des acteurs travaillent le marché à partir de l'entreprise qui tient le rôle de l'offreur mais cherche à maîtriser l'ajustement entre l'offre et la demande.

La restauration collective offre un terrain riche pour saisir les transformations des échanges marchands qui pénètrent les dynamiques productives des organisations et l'activité de travail de ses salariés. Le prestataire de service s'adresse à un client contractuel qui sous-traite la restauration, et à des consommateurs qui tiennent également le rôle d'usager du service. La 
restauration collective révèle l'importance que prend le client dans la dynamique productive de l'entreprise en même temps qu'elle donne à voir les tensions que suscite la prise en compte d'un consommateur usager dans le contact direct du service. La professionnalisation du client, par laquelle l'organisation se saisit du marché, remplace progressivement la personnalisation du client, issue d'un ordonnancement productif où le client pénètre l'organisation.

Les observations sur lesquelles s'appuient nos hypothèses et nos résultats ont été réalisées dans une entreprise qui occupe une position dominante dans le secteur de la restauration collective, et qui peut également être qualifiée de pionnière dans les conduites mises en œuvre pour conquérir ses clients. L'entreprise Restocol permet d'illustrer comment des stratégies d'innovation et de rationalisation peuvent se combiner pour réaliser des ajustements avec les deux figures du client, le client marchand et le consommateur usager.

L'hypothèse centrale qui est ici mise à l'épreuve est celle d'une professionnalisation du client réalisée par les opérateurs du prestataire, en particulier par ses cadres. La professionnalisation en direction du client consiste pour le prestataire à transmettre des savoirs codifiés et les règles de son organisation. En cherchant à faire partager les règles de son organisation, le prestataire attribue également aux clients des identités qui facilitent les arrangements locaux dans un sens défini par lui et plus généralement les ajustements sur le produit, sa qualité et son prix. La mise en forme des clients par l'entreprise se fait d'abord par l'identification des clients et de leurs conditions de satisfaction. Cette identification donne lieu à la construction de catégories qui permet de les labelliser. Sans que la professionnalisation du client soit définie explicitement comme une stratégie du prestataire, une partie importante des conduites de l'entreprise concourt à cette modification des relations avec les clients qui transforment leur identité, que nous définissions comme un processus de professionnalisation.

S'il s'agit de mettre en forme le client (Cochoy et Dubuisson, 2000) pour mieux le maîtriser et se l'attacher, c'est l'organisation tout entière qui est engagée dans ce processus qui renverse la dynamique productive en intégrant le client en amont de ses stratégies. Aux origines de ce processus de professionnalisation du client par l'organisation, c'est un processus de rationalisation de la production qui est à l'œuvre dans l'organisation pour mieux établir son emprise sur le marché. La rationalisation qui a porté dans un premier temps sur les modes de fabrication des repas peut être qualifiée d'industrielle. La rationalisation qui s'est ensuite emparée des relations avec les clients est de type professionnel et a modifié l'ensemble du processus productif. En s'étendant progressivement elle mêle inextricablement rationalisation industrielle et rationalisation professionnelle (Gadrey, 1994).

Le passage de la rationalisation des relations avec les clients à une professionnalisation des clients s'opère lorsque leur sont transmis les critères d'évaluation de la qualité de la prestation. L'entreprise a mis en place des dispositifs pour que les clients s'expriment sur la qualité de la prestation et que leur satisfaction puisse se mesurer, en distinguant les clients contractuels des consommateurs. En maîtrisant ainsi les conditions de leur satisfaction, dans le cadre des dispositifs mis en place par l'entreprise, les clients utilisent dans le même temps les règles définies par l'entreprise. Au-delà de la rationalisation des relations avec les clients qui les posent comme une cible recevant des dispositifs, la professionnalisation reconnaît et s'appuie sur leurs capacités d'acteurs. Dans ce processus de codification (Dubuisson, 1999), le tour de force de la professionnalisation consiste à ce que les clients utilisent eux aussi ces catégories. 
Les opérateurs de cette professionnalisation sont d'abord les cadres. Les responsables de secteur sont une catégorie de cadre particulièrement pertinente pour saisir les mécanismes de ce processus. Gestionnaires de la relation contractuelle, ils ont aussi en charge le management des équipes et des dispositifs sur les restaurants. Nous avons réalisé une observation directe de leur travail en consacrant deux journées à chacun des 40 individus que nous avons accompagnés. Les données sur leur travail au contact des clients et des gérants ont conduit à formuler de nouvelles hypothèses sur la nature du travail des cadres et sur leur rôle dans les échanges marchands.

La professionnalisation des clients participe d'une activité de travail d'encadrement qui consiste à fluidifier les échanges marchands. Cette fluidité marchande poursuit la fluidité industrielle [Vatin, 1987] and [Vatin, 1999]. Il s'agit de s'assurer du bon déroulement des relations commerciales (conditions des transactions, prix) et des relations productives (satisfaction sur le contenu du produit) pour que les destinataires du service soient satisfaits. C'est tout autant une activité en direction des clients qu'en direction des équipes du restaurant. La dimension relationnelle du travail, part entière de l'activité singulière de travail, éclaire ce qu'engage l'acte de travail comme chaînage d'opérations, d'événements, de mise en liens entre personnes, entre personnes et objets, et du coup comme compétences parfois insoupçonnées, comme le met en lumière Anni Borzeix sur le travail d'annonce en gare (Borzeix, 2000). Les compétences «communicationnelles» qui sont mobilisées dans cette activité de travail d'encadrement nous conduisent à supposer l'existence d'une technique des métiers relationnels. Le contenu du travail, tout en étant riche de sens, est technique et finalisé et peut être analysé comme activité technicienne (Vatin, 1999), alors même qu'il porte sur de l'immatériel et du relationnel.

Les clients participent avec les producteurs à la dynamique capitaliste et aux recompositions de l'ordre productif dès les débuts de la société industrielle. Max Weber montre qu'en France, le marché de masse émergea avec la démocratisation des besoins de luxe et détermina l'orientation décisive vers le capitalisme (Weber, 1981, p. 329). Émile Zola nous a livré la fresque romancée de ce qu'ordonne l'organisation d'un grand magasin pour conquérir le «bonheur des dames » (Zola, 1980). La «victoire du marché » interroge les acquis de la sociologie du travail en déplaçant les niveaux de régulation de l'emploi, de l'entreprise et du marché [Reynaud, 1999] and [Segrestin, 2001].

Démonter les mécanismes techniciens de cette chaîne productive participe d'une démarche sociologique qui cherche au-delà des croyances à rendre compte de la matérialité des transformations productives [Dodier, 1995], [Godelier, 1984] and [Vatin, 1999]. Personnalisé dans la figure du client ou naturalisé dans « les contraintes de l'environnement », le lien avec le marché se dissimule plus qu'il ne s'offre au regard du sociologue. La construction de notre objet s'inscrit dans une démarche sociologique hybride. Nous avons fait le pari que le travail d'encadrement opéré avec les clients est un moyen d'appréhender le marché.

Nous montrons dans un premier temps comment Restocol opère progressivement une rationalisation des relations avec les clients, qui produit (deuxième temps de la démonstration) une professionnalisation des clients par l'entreprise, facilitant les ajustements marchands (prix, produit, qualité). 
1. La rationalisation des relations avec le client dans une entreprise de restauration collective ou l'émergence d'un nouvel ordonnancement productif

En intégrant progressivement le client dans ses stratégies (1.1.), l'entreprise s'est dotée de dispositifs de plus en plus perfectionnés qui visent à former le regard du client aux services réalisés par le prestataire. La rationalisation des relations avec les clients soumet la fabrication aux modes de service et estompe inexorablement les frontières entre industrie et service. Les formes des prestations sont renouvelées et les modes de production soumis à un mouvement de normalisation (1.2.).

\subsection{Le client dans la conduite de l'organisation}

\subsubsection{Une spécialisation par segments de clientèles}

Lorsque Restocol au milieu des années 1980 amorce une stratégie de segmentation, en créant trois filiales spécialisées sur les grands clients alors identifiés que sont les établissements de santé, les établissements scolaires et les entreprises privées et publiques, elle s'engage dans une démarche pionnière qui sera reprise près de 10 ans plus tard par ses principaux concurrents. La segmentation s'affinera par la suite, selon des temporalités propres à chaque filiale. Au milieu des années 1990, chacune des trois filiales s'est dotée de structures spécialisées par sous-segments de clientèle. Dans ce qui n'est qu'une première étape dans l'identification de ses clients, Restocol intègre le marché en amont de son organisation en modifiant ses propres structures.

Les découpages effectués avec la segmentation s'adressent autant aux convives qu'aux clients contractuels. Si les utilisateurs sont spécifiés quand personnes âgées et patients hospitalisés sont distingués dans la santé, ce sont les institutions qui sont visées lorsque segment municipal et segment privé se scindent dans le scolaire. Ces deux faces du client ne sont pas pour autant autonomes. Car s'il est nécessaire de distinguer analytiquement et stratégiquement le client contractuel du client consommateur, chacun doit entendre les arguments qui s'adressent aux deux.

La segmentation est associée à un argumentaire spécifique à chaque client qui qualifie également les cadres en relation avec les clients. «Dans le privé, les directeurs d'école sont d'anciens profs, ils ont un côté intellectuel, ils aiment d'autres formes de conversation, d'échange, il faut en face d'eux des gens d'une certaine culture. Le public municipal, ce sont des politiques. L'homme politique est valorisé à travers son interlocuteur » (responsable des ressources humaines, scolaire). Ainsi le client saisi par l'organisation la modifie en retour, de même que ses salariés, qui voient en quelque sorte leur professionnalisation initiale perfectionnée. «La filialisation est une étape clé de l'histoire de l'entreprise en 1984-1985. Les filiales se sont spécialisées par marché. Ça a professionnalisé les structures et les équipes » (directeur des ressources humaines, scolaire).

Si l'entreprise s'adapte à ses clients en les identifiant, elle s'assure également de son emprise sur un territoire dispersé. Les directions régionales qui relayaient en province les politiques de la direction furent démultipliées progressivement avec la filialisation. Le découpage des régions en secteurs achevait de mailler le territoire, et conservait une certaine souplesse à la grande entreprise. La « bureaucratie à clônes » (Gadrey, 1999) se donna les moyens d'avoir la réactivité de «PME dans un grand groupe » (Dondeyne et Gadille, 1999). 


\subsubsection{Transmettre des savoirs codifiés au client}

Confortée par une stratégie innovante qui l'aida à gagner une position de domination, Restocol poursuivit sa conquête des clients en consolidant ses services marketing. En même temps que des savoirs codifiés étaient produits sur les convives et les clients, ils leur étaient transmis, et intégrés dans l'ordre productif de Restocol.

\subsubsection{Par des dispositifs spécifiques}

Forts d'une connaissance enrichie des convives et des clients, les services marketing de Restocol organisent la transmission de ses nouveaux savoirs dans l'entreprise d'une part, et à ses clients d'autre part. À la fin des années 1980 furent élaborés dans la filiale entreprise des outils s'apparentant à des socio-styles destinés à identifier les différents profils des populations fréquentant les restaurants. Ils ont été repris pour être adaptés aux évolutions de ces populations dans chacune des filiales dans les années 1990. La démarche était lancée en 1995 dans la filiale scolaire et deux ans plus tard dans le segment hôpitaux et cliniques de la filiale santé. Les principaux destinataires de ces dispositifs sont les clients contractuels, à qui ils doivent permettre de choisir l'offre la mieux adaptée à leurs besoins.

Désigner les convives est une manière de les inventer (Cochoy, 1999). Si l'identification des types de convives à partir de différences en matière de goût et de consommation alimentaire s'est faite sur des observations donc à partir de l'existant, l'identification peut concourir à la transformation de l'identité en proposant une mise en forme. Les convives désignés dans les socio-styles perdent du même coup leur singularité en s'intégrant dans les catégories du collectif. Ces dispositifs permettent ainsi de réduire l'incertitude liée à l'imprévisibilité des comportements de consommation.

L'identification des conditions de satisfaction des clients et des convives prolonge la recherche d'une maîtrise des comportements des consommateurs. Formalisée dans un dispositif qualité certifié Iso 9002 dans la filiale entreprise en 1995, cette démarche repose sur des principes qui ont déplacé les critères d'évaluation de la qualité du repas vers son environnement. La connaissance du contrat, la qualité de l'assiette, la qualité de l'environnement du repas et du service, l'hygiène et la sécurité alimentaire, la qualité des relations avec les clients et avec les convives définissent formellement la qualité de service. De nombreuses enquêtes de satisfaction existent, adaptées aux types de clients, ciblées sur des points de satisfaction et périodicisées ${ }^{1}$. Elles fournissent au consommateur les critères de son évaluation.

En dernier lieu, le client contractuel s'est vu doté d'un dispositif spécifique pour s'assurer de sa satisfaction. Conçue en 1997 et d'abord mise en œuvre dans la filiale santé, la démarche globale de fidélisation des clients détaille les étapes d'une bonne relation avec le client, du moment de la signature du contrat à sa pérennisation. Une panoplie d'outils et de rendez-vous programmés guide cette gestion contractuelle et relationnelle dont l'ultime étape est de faire savoir au client qu'il est satisfait. "Cette démarche n'a rien de révolutionnaire, elle s'appuie sur des démarches et des outils que tous connaissent déjà chez Restocol. Sa pertinence réside surtout dans la cohérence entre les objectifs et les moyens à mettre en œuvre à chaque étape de la relation avec le client» (responsable marketing, santé). Ainsi innovation et rationalisation professionnelle (Gadrey, 1994) se combinent dans cette démarche qualité. 
En proposant une approche globale du service, intégrant la qualité du repas, mais aussi son environnement et le respect du contrat, Restocol formalise pour le client les actions menées à son intention. En mettant à jour toutes les dimensions du service, l'organisation démontre que son professionnalisme repose sur l'ensemble de ces actions, et appelle le client à reconnaître que la satisfaction sur une prestation de restauration repose sur un ensemble de savoirs et d'actions qui débordent le strict cadre de la qualité du repas.

La transmission de ces savoirs codifiés au client et aux convives se réalise également par de véritables programmes de formation. Des diététiciennes (les hommes ne sont pas encore engagés dans ce rôle) et des responsables qualité ont pour mission, outre de former en interne le personnel de Restocol, de rencontrer les clients pour les former aux règles de nutrition et d'hygiène alimentaire. En scolaire, ces spécialistes rencontrent des parents d'élèves et des groupes d'enfants. En santé, les aides-soignantes de l'établissement client qui sont impliquées dans le service des repas sont davantage les destinataires de ces formations. L'entreprise ne néglige aucun de ses interlocuteurs qui composent une figure complexe du client.

Le rendez-vous annuel de l'innovation organisé par Restocol permet de diffuser tant dans l'organisation qu'aux clients actuels et potentiels l'ensemble des démarches particulières engagées dans les différents segments ou dans des sites pilotes. La formalisation que requiert la présentation d'une innovation à ce rendez-vous prisé et primé contribue à codifier davantage les savoirs sur lesquels reposent les innovations, quel que soit leur degré, et facilite leur généralisation.

\subsubsection{Par des prestations qui révèlent ces savoirs}

Au-delà de dispositifs spécifiques, les prestations et les services offerts aux clients révèlent l'existence de ces savoirs codifiés et l'élargissement des critères de la qualité du service.

Les formules de restauration s'appuient sur les enquêtes qui permettent d'établir les tendances en matière de goût de la population qui fréquente les restaurants. La gamme des prestations propose une cuisine du terroir, une cuisine nature ou encore une cuisine du monde. Les offres culinaires se diversifient et rafraîchissent l'univers des cantines uniforme et monotone. Les cantines au menu unique paraissent maintenant archaïques face aux boutiques et aux enseignes dans lesquelles les convives font leurs choix. La restauration s'adapte aux goûts des convives et aux besoins nutritifs divers. Des nourritures roboratives seront proposées dans le restaurant d'un site industriel fréquenté majoritairement par des ouvriers tandis que des salades variées à composer soi-même apparaîtront sur les comptoirs des sites où se sustente une population féminine soucieuse de sa ligne.

L'offre culinaire est mise en scène dans des stands qui associent le plat, le service et l'architecture. La restauration est abordée comme un concept par les hommes du marketing et le restaurant doit mettre en valeur les repas. Quand les buffets et les enseignes remplacent le self-service linéaire, le convive est guidé tout au long du parcours pendant lequel il compose lui-même son assiette.

Parallèlement à ces innovations sur les prestations de restauration, Restocol élargit les services de la restauration au cadre de vie d'une collectivité. Le nettoyage, l'entretien des espaces verts, et plus généralement la prise en charge globale de l'infrastructure du client apparaissent progressivement dans la gamme de services proposés depuis le début des 
années 1990. Les services d'hôtellerie sont apparus au départ dans la filiale santé, et la prise en charge globale de client se développe dans la filiale entreprise. Restocol répond ainsi aux besoins de sous-traitance de ses clients qui se recentrent sur leur «cœur de métier» en déléguant les activités annexes.

\subsection{La progressive normalisation de l'organisation de la production}

En même temps que les offres de Restocol se diversifient, l'organisation de la production se normalise progressivement et intègre l'évolution de l'offre. Le client a ainsi pénétré en amont du processus de production, les services marketing participant à la sélection des produits avec le service achat. Ainsi un responsable des achats nous a expliqué le sens de l'évolution de la fonction achat chez Restocol: "C'est une démarche en collaboration avec le service marketing : optimiser la qualité perçue par la qualification et la sélection des produits. » Le service marketing détermine des critères de qualité perçue par les clients et sélectionne les produits qui répondent à ces critères. Des fiches techniques de produits sont alors élaborées et transmises au gérant qui doit les utiliser. «La conception des fiches par le service marketing se base sur des catégories de convives, et définit des odeurs et des goûts pour chaque plat. Le service achat imagine des solutions de produits répondants aux critères, puis les teste, en fonction de la représentativité des restaurants. Le service achat élaborera alors une fiche technique produit, avec les modes de conditionnement, de conservation... Le service achat demande aux interlocuteurs des achats amont de lui proposer des produits conformes au cahier des charges constitué par la fiche technique produit achat. La démarche est alors inversée : ce n'est plus Restocol qui choisit par rapport à ce que lui propose le fournisseur, c'est Restocol qui exprime un besoin précis. Ce fonctionnement permet d'accéder à de nouvelles gammes qui n'étaient, jusque-là, pas retenues spontanément par les distributeurs pour la restauration collective. La priorité sera accordée dans chaque filiale aux produits auxquels les convives sont sensibles, complexes à cuisiner, et représentant un volume d'achat important. L'objectif est qu'à trois ans cette démarche soit appliquée à $75 \%$ de notre volume achat $\gg$ (responsable direction achat).

On voit ainsi à quel point la gestion des relations avec les clients a conduit à la recherche de nouvelles solutions dans l'organisation pour améliorer la qualité du service rendu, entraînant des phénomènes d'innovation et de rationalisation. Le processus de rationalisation, qui pénètre alors l'organisation Restocol et les relations avec les clients, s'appuie sur la mise à jour de ces nouvelles connaissances, et il est ainsi indissociable d'une codification des savoirs portant sur les clients et leurs conditions de satisfaction. La mise en place de nouvelles règles configure un nouvel ordonnancement productif.

C'est cependant un processus qui avance pas à pas, de manière parfois chaotique et fragmentée. Cette restauration collective rénovée qui se rapproche de la restauration commerciale et de la grande distribution se diffuse de manière éparse et discontinue à l'intérieur des différents segments de clientèle. La justification du nouvel ordonnancement productif entraîne un processus de légitimation de la rationalisation.

\section{La professionnalisation du client ou comment partager les règles de l'organisation}

\subsection{De l'hypothèse d'une socialisation au restaurant à l'hypothèse d'une professionnalisation} du client par l'organisation 
Une des hypothèses que nous formulons est que dans les interactions répétées entre les clients et les interlocuteurs du prestataire s'opère un processus de socialisation. Le consommateur, le client et les interlocuteurs du prestataire s'attribuent aux uns et aux autres des identités dont l'effet objectivant va marquer progressivement leur appartenance à tous au petit monde du restaurant, sorte de zone franche entre Restocol et l'établissement client. La «mama des écoles » est l'archétype peut-être le plus évocateur de ces identités attribuées, mais il en existe d'autres pour qualifier chacun des protagonistes.

La restauration collective est une activité du quotidien. Servir tous les jours des repas aux mêmes consommateurs, sur les lieux de travail, à l'école ou dans des maisons de retraite instaure une proximité entre les acteurs de l'organisation et les convives du restaurant, intégré à la vie de l'établissement du client marchand. D'une certaine manière, Restocol rentre dans l'espace privé et domestique du client, avec la restauration, mais également quand elle élargit les services rendus au nettoyage, ou aux services à l'immeuble. Le client tient souvent le rôle d'un deuxième patron pour les employés du restaurant salariés de Restocol. Bien souvent, le référent des pratiques pendant la pause du repas s'apparente au monde domestique, dans cet entre-deux que les convives ne passent pas à la maison mais qui leur permet de s'échapper d'un univers parfois contraignant.

L'histoire de l'entreprise racontée par les dispositifs qu'elle a mis en place suggère de distinguer deux époques dans ce processus de socialisation que l'on peut qualifier de mutuel, puisqu'il concerne deux institutions, celle de Restocol, et celle du client. Pendant la première époque une socialisation de Restocol au client est majoritairement à l'œuvre, qui correspond à un service personnalisé associé à une production dont les modes tendent à s'industrialiser. Les étapes de production et de service peuvent ainsi être distinguées jusqu'au début des années 1990. Ensuite s'amorce une deuxième époque dans laquelle la normalisation couplée de la production et du service se met progressivement en place. Production et service deviennent de plus en plus étroitement imbriqués, impliquant un travail de gestion des interdépendances de plus en plus complexe. Le client remonte dans l'organisation (Cochoy, 1999), dans le sens où en amont du processus de production, les dispositifs sont pensés pour lui, mais ce faisant Restocol met en forme le client. Cette mise en forme a requis l'élaboration progressive d'une catégorisation des clients marchands et des consommateurs et a abouti à leur typification (Gadrey, 1994). Ce «labelling» du client produit par Restocol lui attribue une identité et donne lieu à une transaction objective et synchronique, produite par le jeu-dont Restocol maîtrise mieux qu'auparavant les règles - de l'offre et de la demande dans un espace et un temps ponctuels.

Pour qu'il y ait effectivement une socialisation du client à l'organisation, et non une socialisation mutuelle au restaurant, il faut qu'un ensemble de règles manifeste de manière explicite l'appartenance du client en tant que tel à l'organisation productive. Ce qui détermine le client va s'objectiver de plus en plus, au fur et à mesure de son intégration dans les instances de l'organisation au cours de sa socialisation, favorisant une réduction du sens des valeurs en direction d'un consensus (Dehier, 1997). La professionnalisation vient donc réorienter le processus de socialisation dans le restaurant en renversant le sens de la transmission des règles. L'enjeu de la professionnalisation pour le prestataire est d'attribuer aux clients des identités qui facilitent les arrangements locaux dans un sens défini par lui. Il transmet ainsi plus aisément les règles de son organisation aux clients, ou du moins, il en rapproche les compromis qui se trouvent dans les ajustements avec les clients. À partir du 
moment où le client utilise les catégories définies par Restocol qualifiant les consommateurs et que ceux-ci s'y reconnaissent, la professionnalisation est à l'œuvre.

La professionnalisation du client organisée par Restocol recouvre en réalité un double travail, l'un en direction des clients marchands, l'autre en direction des consommateurs. En effet, les identités attribuées à ces deux figures du client sont distinctes, et les ajustements auxquels Restocol veut procéder dans la transaction portent l'un sur l'aspect contractuel des relations marchandes, l'autre sur le mode de consommation dans la relation de service. Il ne s'agit pas de transmettre les mêmes règles à ces deux figures du client. Les règles qui concernent le client marchand portent sur la définition de la prestation globale réalisée par Restocol : quels sont les services pris en charge, quel type de restauration, sous quelles modalités contractuelles (forfait, assistance technique...). Les règles qui concernent le consommateur sont davantage circonscrites au moment du service : orientation dans ses choix, quantités et qualités des plats, parcours de la salle du service à la salle à manger pour faciliter le déroulement du service. On a vu que les enquêtes de satisfaction contenaient les critères de jugement sur la qualité de service. Ces règles doivent ainsi établir la satisfaction du consommateur et du client marchand.

Le processus de professionnalisation pose l'interlocuteur contractuel comme le véritable client. Les opérateurs de la relation avec le client ne sont pas les mêmes selon qu'il s'agisse du client marchand ou du consommateur. Si le responsable de secteur s'adresse de manière privilégiée au client marchand, le travail de management effectué sur le gérant et l'équipe du restaurant vise indirectement la relation avec le convive. Le gérant quant à lui doit gérer la double relation. L'équipe du restaurant est dévolue aux convives. Cependant les ajustements qu'il opère avec le client marchand portent davantage sur un registre technique (Gadrey, $\underline{1994)}$ alors que ceux gérés par le responsable de secteur portent sur le registre contractuel et marchand.

Bien que les compétences attribuées par Restocol au gérant, notamment en matière de gestion, tendent à s'élargir, et que le gérant devienne un interlocuteur sérieux pour le client, le prestige du cadre honore le client. Comment et à quelles conditions s'opère cette professionnalisation dans le travail quotidien de ses opérateurs, quand elle fonctionne ? Les illustrations que nous proposons témoignent d'un degré d'avancement différencié.

\subsection{Transmettre les règles de l'organisation au client}

La finalité du travail en contact avec le client est de transformer la demande du client, pour qu'elle soit solvable par l'organisation tandis que la finalité du client, quand il s'adresse à l'organisation, est de transformer l'offre de l'organisation, pour qu'elle s'adapte à sa demande. Les tensions naissent alors de ce que des compromis sont à faire quand les ajustements renferment des contradictions potentielles.

Nous voulons tester ici l'hypothèse que le travail des responsables de secteur consiste à faciliter la fluidité des échanges marchands. Il s'agit d'appréhender «l'acte de travail comme l'accès pragmatique du monde présent aux mondes possibles de production » (Salais, 1992), l'activité de travail des cadres (pour) suivant la recherche menée par Restocol d'une extension de la fluidité industrielle à la fluidité économique, où les transactions en continu poursuivent la voie d'une production en continu. 
2.2.1. Quand la professionnalisation du client marchand gagne du terrain: le travail des responsables de secteur

Les responsables de secteur sont garants de la qualité des prestations et travaillent dans l'organisation en transmettant et en contrôlant l'application des politiques de l'entreprise par les gérants des restaurants et leurs équipes. Ils sont également garants de la satisfaction des clients et travaillent (dans) le marché en gérant la relation avec les clients. Outre la responsabilité des résultats budgétaires des restaurants, les responsables de secteur mettent en place les dispositifs visant la qualité de service, qui portent en premier lieu sur la satisfaction des convives et la qualité des repas, et s'étendent à la gestion des relations avec les clients institutionnels. Si le responsable de secteur est un gestionnaire et ne vend des nouvelles prestations qu'aux clients qu'il a déjà ${ }^{2}$, il est vendeur en étant représentant de son organisation et de ses produits, et en démontrant que des prestations innovantes, diversifiées et plus sûres, sont proposées par Restocol.

L'importance du travail d'argumentation opéré par les responsables de secteur nous a conduit à poser l'hypothèse d'une technique des métiers relationnels. La performation [Cochoy, 1999] and [Borzeix, 1987] opérée par la parole en acte suggère de reprendre les pistes du travail sur les effets des «faits de langue comme des faits sociaux » (Borzeix, 1987) pour explorer l'activité de travail des cadres. Sur quels ressorts cachés s'appuie le travail de management ? Le travail d'argumentation, n'est-il pas aussi le prolongement de postures et de gestes, le corps recelant les croyances les plus intimes, incitant à observer la socialisation comme un processus construit dans l'espace et le temps, mais aussi dans les corps (Haicault, 2000) ? Nous nous bornons ici à observer ce travail d'argumentation comme travail de justification et travail d'occultation. L'observation directe a consisté à accompagner les responsables de secteur pendant deux journées de travail chacun, au cours desquelles étaient prévus des rendez-vous avec des clients et des rendez-vous avec des gérants. Des explications nous étaient données avant et après pour bien définir les situations observées sans les perturber. Cette immersion dans le terrain se faisait pendant un mois que nous avons passé dans une direction régionale, en faisant le tour de tous les cadres. Nous avons répété l'opération trois fois, dans trois régions et auprès de trois types de clients différents. Nous proposons au lecteur des récits qui illustrent le processus de professionnalisation, pour en dégager ensuite les conditions de fonctionnement.

La démonstration orchestrée par Sébastien, responsable de secteur et Sabine, responsable marketing pour présenter un projet d'agrandissement de la cafétéria au client de Sébastien, M. Agostini, directeur de la clinique Saint-André dans le Var, a nécessité plusieurs réunions de préparation avant le rendez-vous. La présence de Sabine donne un caractère rituel à la rencontre. Elle fait figure de spécialiste, et parle des réalisations de Restocol dans d'autres établissements prestigieux de la région. Elle témoigne ainsi de l'expérience reconnue de Restocol et de ses compétences. Elle rassure également M. Agostini sur la disponibilité des interlocuteurs de Restocol sur ce projet particulier. Sébastien pendant ce rendez-vous parle du prestige de Saint-André, qu'il connaît bien, et des bénéfices que M. Agostini peut espérer, alors que les visiteurs à l'heure actuelle sont contraints d'aller à l'extérieur faute d'une capacité d'accueil suffisante de la cafétéria. Sabine donne des arguments plus techniques sur la décoration, les matériaux, les couleurs, les délais des travaux. En même temps, Sébastien en profite pour faire le point sur le contrat, et souligne le rôle d'accueil auprès des visiteurs que jouent le gérant et l'employée de service qui tient la cafétéria. Ils ont planifié de concert la réalisation du projet, afin que le client soit prêt à accepter toutes leurs propositions. Peu de 
place est laissée au hasard pendant ce rendez-vous, Sabine et Sébastien interviennent au moment le plus opportun pour appuyer leurs arguments respectifs. Les registres du discours, les postures et les gestes sont adaptés à chaque étape pour correspondre à la tonalité du moment, instaurant tour à tour un caractère cérémonial pour commencer l'entretien, sérieux au cœur de la démonstration, et convivial pour clôturer la rencontre. Le déjeuner et le ton plus détendu qui y prévaut confirment que Sébastien est l'interlocuteur privilégié de M. Agostini et que des relations cordiales règnent entre Restocol et le client. Il suit la relation en continu, en utilisant les nouveaux dispositifs (reporting, enquêtes périodicisées sur la satisfaction...). Sébastien annonce le souhait du directeur régional de Restocol de rencontrer M. Agostini, voulant s'assurer en personne de sa satisfaction. Ainsi, le responsable de secteur mobilise l'organisation Restocol tout entière pour convaincre son client. En ce sens, professionnaliser le client, c'est vendre l'organisation.

La mise en place des prestations innovantes et des nouveaux dispositifs a accru les besoins de coordination entre les responsables de secteur et les cadres fonctionnels. Les responsables marketing sont sollicités pour l'amélioration des prestations existantes ou la mise en place de nouvelles prestations (repas ou autres services), les responsables « ressources humaines» pour les besoins de formations que nécessitent les plans d'action qualité de service, les diététiciennes ou les responsables qualité, pour des actions ponctuelles sur la formation par exemple. Ces coordinations, si elles sont rendues nécessaires par les nouveaux dispositifs, et parfois vécues par les responsables de secteur sur le mode de l'imposition, sont en même temps un moyen pour le responsable de secteur de légitimer son action auprès du client.

La présence de nombreux interlocuteurs cadres permet d'honorer le client et les rapports sociaux participent de cette répartition des rôles entre le gérant, le responsable de secteur et les cadres fonctionnels. Cependant la technique et les savoirs ne sont pas absents dans la gestion de la relation avec le client. Ainsi Sébastien fait confiance à Joseph, le gérant de l'établissement de Saint-Albert pour régler avec le client une partie des problèmes liés à l'organisation du nettoyage que Restocol vient de signer sur cet établissement. Par contre il sait Robert capable de commettre des impairs sur Saint-Christophe et lui délègue moins de responsabilités. Il faut être capable d'identifier les interlocuteurs pertinents, d'adopter le ton juste avec chacun d'entre eux, et de soulever les problèmes ou signaler les réussites aux moments opportuns.

Dans un processus de production où les repas industrialisés nécessitent moins d'interventions directes des hommes (perte des savoir-faire de cuisiniers, des «tours de main » diront certains) mais tout autant d'attentions (cuissons, quantités) et de savoirs techniques et gestionnaires, le travail du responsable de secteur assure la fluidité de la chaîne productive étendue pour inclure les clients.

La formalisation des actions permet la démonstration d'un travail d'organisation. Bertrand, responsable de secteur du restaurant de l'entreprise Soulier, a profité de la mise en place d'une prestation nettoyage pour «mettre à plat » avec le gérant Fabien, l'ensemble des menus services réalisés par Restocol sur l'établissement, que l'habitude finit par rendre invisible. Ces actions quotidiennes que Fabien considère comme naturelles, il faut pour le responsable de secteur les mettre en valeur, car elles deviennent indispensables pour le client. Ainsi l'objectivation d'actions menées par le gérant sur un mode informel et personnalisé permet de les rendre apparentes. Pour organiser le nettoyage sur le restaurant, le binôme a convoqué également le responsable qualité, qui a formé le regard du client aux aspects hygiène, qualité 
et sécurité. De cette manière, le client est amené à reconnaître l'ensemble du travail réalisé par Restocol. Il s'agit de rendre visible l'invisible, de démontrer tous les détails d'un travail d'organisation que requiert le service. Le passage du client personnalisé au client normé conduit à réduire l'incertitude, mais également à identifier des besoins qui, débordant le seul cadre de la restauration, n'auraient pas été visibles sans la formalisation.

Tandis que la connaissance des clients et de leurs conditions de satisfaction quant à la qualité du service rendu s'améliore et produit des savoirs codifiés, la normalisation gagne du terrain en systématisant les spécificités reconnues. Ainsi, si la normalisation est symptomatique d'une montée du client dans l'organisation (Cochoy, 1999), elle s'impose progressivement aux responsables de secteurs et aux gérants qui, quand ils sont livrés à eux-mêmes, personnalisent les services rendus aux clients.

Au-delà de ces composantes matérielles du travail qui voient les actes et les coordinations se multiplier avec la complexification des dispositifs à mettre en place, une grande part du travail d'encadrement des responsables de secteur est un travail de justification, tant en direction des clients, qu'en direction des gérants qu'ils managent dans la mise en place des nouveaux dispositifs sur les restaurants.

Le sens donné aux actions des responsables de secteur et des gérants se modifie au fur et à mesure que le client est intégré en amont: il s'agit de le réintroduire dans la plupart des actions entreprises dans l'organisation. Le responsable de secteur ne passe pas forcément plus de temps avec un client normé qu'avec un client personnalisé. Mais pour le client normé, tout travail peut être considéré comme étant destiné au client, alors que pour le client personnalisé, les responsables de secteur et les gérants ont tendance à considérer uniquement le temps passé en contact avec le client comme travail pour le client. C'est donc bien une rationalisation du client qui est à l'œuvre, rationalisation de la relation d'abord, puisqu'il s'agit de montrer que les relations directes et de contact ne sont pas les seules destinées au client, rationalisation des temps également, décomposés en autant d'actions que l'on rapporte au client. La formalisation des actions qui s'adresse au client permet de rapporter toutes les actions entreprises à la satisfaction de ce dernier. Le travail dans l'organisation prend son sens dans le travail du marché. Il ne disparaît pas bien au contraire, car ce sont tout autant de coordinations dans l'organisation qui sont à repenser en fonction du client, et qui peuvent s'en trouver accrues.

C'est également un travail d'occultation, qui réussit à faire disparaître les modes de production en mettant l'accent sur la prestation globale. Pendant toutes nos observations avec des clients, il n'a pas été question d'aborder les modes de production des repas, les achats de denrées qui pourraient pourtant être considérées comme essentielles à la qualité du service dans un secteur de la restauration. La formalisation des actions et le travail d'occultation sont également au principe du travail de management qui gomme la perte de métier de cuisinier chez les gérants pour dessiner un professionnel plus gestionnaire. Un des tours de force de ce travail d'occultation est d'avoir réussi à faire disparaître des acteurs agissants dans la relation de service. Les employés de service, en grande majorité des femmes, souvent à temps partiel, comptent pour près de la moitié de la population de l'entreprise, et pourtant elles sont très peu évoquées dans les actions menées. Les raisons de cette disparition sont plurielles. Ce qui nous intéresse ici c'est que ce double travail de justification, vers les clients et vers les équipes, permet en éludant les problèmes possibles et en soulignant les actions positives de légitimer progressivement la professionnalisation du client telle qu'elle est organisée par l'entreprise. 
La figure du client se transforme progressivement dans ce processus. Au départ il sous-traite la restauration dans son établissement. Mesurés sous «plusieurs coutures », ses besoins sont analysés comme évolutifs, et de nouveaux aspects de son organisation sont identifiés et pris en charge. De nouvelles images des clients se révèlent et agissent. Il devient plus professionnel et plus exigeant, en même temps qu'il devient progressivement client de l'organisation : il bénéficie du savoir-faire de Restocol dans son entier, et non plus seulement des services rendus par le gérant et son équipe sur le restaurant.

\subsubsection{Les employés de restaurant, entre le client et l'entreprise de restauration}

Nous avons également réalisé un travail d'observation participante en tant qu'employée de service dans trois restaurants de taille et de type différents, pendant des périodes de trois semaines. Il est apparu que les équipes du restaurant étaient inégalement concernées par les dispositifs de Restocol à destination des consommateurs. Le service nécessite de résoudre des problèmes de quantités à servir et de files d'attente. Les normes de l'organisation imposent un grammage, souvent dépassé si le convive le demande. Les employés de service ne se considèrent pas toujours comme les employés de Restocol, mais parfois comme les employés du client ${ }^{\underline{3}}$. Pour ceux, le plus souvent cuisiniers, qui se considèrent comme employés de Restocol, se fait un arbitrage, selon avec qui ils font le service, la fréquence des demandes, et la compensation possible avec des gens qui prennent des plus petites assiettes. Pour éviter la queue, il faut aller vite, ce qui demande technicité et habileté, pour bien présenter l'assiette. Une bonne disposition permet de rendre l'assiette plus appétissante et de donner également l'impression qu'elle est plus pleine. Mais la convivialité demande de ne pas aller trop vite non plus et de servir chaque personne avec le sourire, même si 3000 personnes sont servies. Préparer trop d'assiettes à l'avance, c'est les laisser refroidir, et ne pas voir passer tous les convives qui se saisiront simplement de l'assiette, et auront eu le service rapide sans le sourire.

En tant qu'employée de service et sociologue stagiaire pour l'occasion, nous avons participé dans un grand restaurant de la région parisienne à la mise en place d'une enquête de satisfaction et à son dépouillement. C'est le personnel du restaurant qui doit aller enquêter, car l'enquête de satisfaction est aussi un outil de gestion des ressources humaines à destination du personnel, afin de le sensibiliser à la satisfaction des convives. Le premier enseignement a été celui des problèmes de maîtrise de la langue et de l'écrit de nombreux employés pour poser les questions et pour la prise de note nécessaire à l'enquête. Il vaut mieux ne pas déranger les convives quand ils mangent, alors que l'enquête ne peut se réaliser que pendant le temps du repas. Le dépouillement de l'enquête a montré que les insatisfactions portent souvent sur des problèmes liés au cadre du repas : une salle de restaurant trop bruyante, des plateaux encore humides, une queue trop importante à telle heure. La prestation relativement haut de gamme délivrée et le choix offert sur ce restaurant laissaient peu de place aux récriminations sur les repas eux-mêmes.

\subsection{Conclusion : les conditions d'une professionnalisation des clients}

À quelles conditions le client marche-t-il dans l'organisation quand celle-ci organise le marché ? Les résultats de nos observations confirment que si un processus de professionnalisation des clients est bien à l'œuvre dans l'entreprise Restocol, il se diffuse de manière inégale et parfois chaotique chez les clients. Le client ne marche pas toujours dans 
l'organisation. En ce sens, il s'agit bien d'une professionnalisation organisée par Restocol, conduite ordonnée qui ne présuppose pas de sa réussite.

Du côté des opérateurs de Restocol, l'activité de travail des responsables de secteur est nécessaire pour que la professionnalisation des clients contenue dans les dispositifs de l'entreprise s'opère. La démonstration rapportée au client du travail de l'organisation s'appuie sur la formalisation et la codification qui repose sur des savoirs et des techniques, en même temps que sur un travail de justification. Cependant les responsables de secteur n'échappent pas aux phénomènes d'appropriation du changement organisationnel, et une partie se retrouve davantage dans l'ordre de la personnalisation qu'ils associent à une plus grande autonomie. L'ordre normé accroît pour ceux-là la charge administrative du travail et fait courir le risque de la bureaucratie à la grande entreprise. Le rapport social rattaché à la catégorie cadre participe également au processus de professionnalisation effectué par les responsables de secteur.

Les employés du restaurant sont partagés entre les règles de Restocol et celles du client, et optent encore souvent pour ces derniers, ce qui s'explique par une socialisation plus forte dans l'établissement du client, ainsi que par une gestion de l'emploi qui s'appuie sur une flexibilité taylorienne (Gadrey, 1999) que l'on retrouve également dans la grande distribution alimentaire (Guélaud et al., 1989) (faibles niveaux de rémunération, emploi à temps partiel contraint, associé à de faibles niveaux de qualification).

Existe-t-il du côté du client des prédispositions à la professionnalisation ? Le repérage de clients contractuels plus ouverts à la professionnalisation semble suivre l'arbitrage entre le prix de la prestation et sa qualité, la professionnalisation étant plus aisée à mettre en place dans les prestations les plus innovantes. Cependant, les entretiens avec les clients contractuels que nous avons rencontrés montrent qu'ils encouragent cette évolution de la relation marchande, qui leur donne à leur sens un plus grand contrôle de la prestation, et assure une plus grande confiance dans le prestataire. La diversification des critères repérée dans les appels d'offre pourrait alors encourager cette tendance.

Le client est-il manipulé dans un processus d'innovation et de rationalisation initié par Restocol, ou bien le client est-il roi, son exigence et son instabilité appelant sans cesse des transformations des prestations et de l'organisation qui lui fournit le service ? La codification du comportement des consommateurs qui participe à la professionnalisation des clients peut susciter la crainte de la «Mac Donaldization» décelée par Georges Ritzer (Livian, 2000). Dans le cadre de la restauration collective, les consommateurs sont en quelque sorte prisonniers dans la relation triangulaire entre eux-mêmes, le prestataire, et le client contractuel. Ils suivent l'organisation quotidienne du service de Restocol. Dans le cadre ponctuel des enquêtes de satisfaction se décelait le souci de ne pas mettre en cause les employés de restauration, en émettant des critiques portant le plus souvent sur l'environnement du repas, ce qui finalement encourage la conduite de Restocol qui élargit les critères de satisfaction sur la prestation.

La thèse d'une professionnalisation du client conduite par l'organisation participe d'une conception des relations entre l'organisation et le marché où les deux s'alimentent l'un et l'autre. Si les règles que l'organisation cherche à partager avec le client sont source de contrainte, elles créent aussi de la confiance [Reynaud, 1997] and [Karpik, 2000]. Par ce double effet des règles, le client est conduit à les adopter et à rentrer dans les normes de 
l'organisation, mais il en tire également profit. On pourrait également entrevoir que les relations contractuelles, comme institutions du marché, sont en passe d'être refondées en renouvelant les formes de l'apprentissage réciproque des comportements de transaction (Williamson, 1994).

Références

Borzeix, 1987 A Borzeix, Ce que parler peut faire. Sociologie du travail, 292 (1987), pp. $157-176$.

Borzeix, 2000 A Borzeix, Relation de service et sociologie du travail. L'usager : une figure qui nous dérange ?. Cahiers du genre, 28 (2000), pp. 19-48.

Cochoy, 1999 F Cochoy, Une histoire du marketing, discipliner l'économie de marché, La Découverte, Paris (1999).

Cochoy et Dubuisson, 2000 F Cochoy and S Dubuisson, Introduction. Les professionnels du marché : vers une sociologie du travail marchand. Sociologie du travail, 423 (2000), pp. $335-359$.

Dehier, 1997 G Dehier, Individu et société. Détermination et indétermination, J.P Durand, R Weil, Editors, Sociologie contemporaine, Vigot, Paris (1997), pp. 319-335.

Dodier, $1995 \mathrm{~N}$ Dodier, Les hommes et les machines, La conscience collective dans les sociétés technicisées, Métailié, Paris (1995).

Dondeyne et Gadille, 1999 C Dondeyne and M Gadille, The Effects of the Multidivisional Form of Modern Corporation on Workers Behaviors and its Effectiveness: a Challenge for the Societal Effect Theory? Interim conference: Economic Sociology at the edge of the 3rd millenium, janvier, (1999).

Dubuisson, 1999 S Dubuisson, Les recettes du restaurant. La codification comme moyen de créer des opportunités d'action. Sciences de la société, 46 (1999), pp. 1-14.

Gadrey, $1994 \mathrm{~J}$ Gadrey, La modernisation des services professionnels. Rationalisation industrielle ou rationalisation professionnelle ?. Revue française de sociologie, 352 (1994), pp. 163-195. 
Gadrey, 1999 J Gadrey, Flexibilité et professionnalisation du travail dans les services : des stratégies et des modèles distincts. Économies et sociétés, 1-5 (1999), pp. 117-141.

Godelier, 1984 M Godelier, L'idéel et le matériel, Fayard, Paris (1984).

Guélaud et al., 1989 F Guélaud, C Lanciano, M Lemaire, G Roustang and F Sellier, La flexibilité du travail dans les hypermarchés, Rapport de recherche, Lest-CNRS, Aix-enProvence (1989).

Haicault, 2000 M Haicault, L'expérience sociale du quotidien. Corps, espace, temps, Les Presses de l'université d'Ottawa, Ottawa (2000).

Karpik, 2000 L Karpik, Le guide rouge Michelin. Sociologie du travail, 423 (2000), pp. $369-389$.

Livian, 2000 Y.F Livian, Compte rendu de l'ouvrage de Georges Ritzer, The Mac Donaldization thesis, Sage, Londres, 1998. Sociologie du travail, 423 (2001), pp. 512-514.

Reynaud, 1997 J.D Reynaud, Les règles du jeu. L'action collective et la régulation sociale, Armand Colin, Paris (1997).

Reynaud, 1999 J.D Reynaud, Communication au colloque des quarante ans de sociologie du travail, Table ronde des fondateurs, 25-26 novembre, (1999).

Salais, 1992 R Salais, Modernisations des entreprises et Fonds national de l'emploi. Une analyse en termes de mondes de production. Travail et emploi, 51 (1992), pp. 49-69.

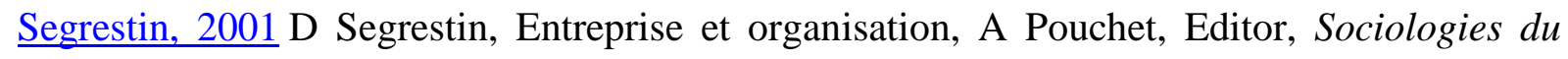
travail : quarante ans après, Elsevier, Paris (2001).

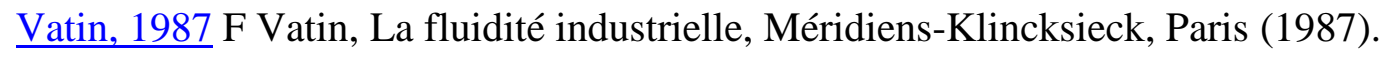

Vatin, 1999 F Vatin, Travail, sciences et société, Éditions de l'université de Bruxelles, Bruxelles (1999).

Weber, 1981 M Weber, Histoire économique. Esquisse d'une histoire universelle de l'économie et de la société, Gallimard, Paris (1981), (édition originale, 1923). 
Williamson, 1994 O.E Williamson, Les institutions de l'économie, InterÉdition, Paris (1994).

Zola, 1980 É Zola, Au bonheur des dames, Gallimard, Paris (1980), (édition originale, 1883).

\section{Auteur correspondant}

${ }^{1}$ Sophie Dubuisson a fourni une description sur ce point précis, montrant comment, même pour les enfants, sont trouvées des solutions pour évaluer et améliorer leur satisfaction (Dubuisson, 1999).

${ }^{2}$ Le responsable de développement vend des prestations à des nouveaux clients.

${ }^{3}$ Un dispositif législatif garantit la reprise du personnel du restaurant lorsque le prestataire de service change. Pour ces employés de service, ce sont les employeurs qui se succèdent, mais le client reste le même. 